\title{
Relaxation approach for the generation of inhomogeneous distributions of uniformly sized particles
}

\author{
J. Hunt, ${ }^{\text {a) }}$ N. Kundtz, N. Landy, and D. R. Smith \\ Electrical and Computer Engineering, Duke University, 130 Hudson Hall, Durham, \\ North Carolina 27706, USA
}

(Received 15 April 2010; accepted 8 June 2010; published online 15 July 2010)

\begin{abstract}
For many applications in gradient index devices and photonic crystals, it is necessary to be able to design semicrystalline distributions of particles where the lattice constant of the distribution is an arbitrary function of position. We propose a method to generate such distributions which is physically motivated by a system of interacting particles, and apply it to the design and implementation of a microwave gradient index lens. While the demonstration was preformed at microwave wavelengths, this technique would also be particularly useful for designing devices for operation at IR and visible wavelengths where the fabrication of distributions of uniformly sized holes or columns is very easy. (C) 2010 American Institute of Physics. [doi:10.1063/1.3460914]
\end{abstract}

For many applications it is useful to have a twodimensional (2D) crystalline distribution of inclusions, usually holes or columns in a dielectric matrix, with some spatial variation in the lattice constant of the distribution. For example, gradient index (GRIN) metamaterials may be designed using distributions of inclusions in a dielectric by employing an appropriate effective medium approximation. These distributions should be crystalline because the effective medium is then defined over the smallest possible length scale, one unit cell, and Rayleigh scattering is minimized as compared to an amorphous distribution. ${ }^{1}$ In addition, these distributions should have hexagonal symmetry since this allows for the largest range of achievable indices and good isotropy. ${ }^{2}$ This is a particularity useful method for fabricating devices operating at IR or visible wavelengths because uniformly sized holes or columns in a dielectric are easy to make with precise positioning. Hole/column distributions have been used to create IR carpet cloaks ${ }^{3,4}$ and microwave luneburg lenses ${ }^{5}$ among other things. Photonic crystals (PCs) with spatially varying lattice constant ${ }^{6,7}$ and graded index ${ }^{8}$ have also been studied. By spatially varying the lattice constant in a PC, the bandstructure can be spatially varied smoothly. These structures may have applications in waveguide couplers, ${ }^{9}$ slow light optical buffers, ${ }^{10}$ and high-Q lasers. ${ }^{11}$

However, previous implementations rely on characteristics unique to each design to generate the inclusion distribution. The distribution may be generated by applying a transformation to a regular grid and placing an inclusion at each grid point, ${ }^{3}$ however, this method requires you start with a known grid and can find a transformation that will yield the desired lattice constant at each location which is not possible in general. Alternatively, inclusions may be evenly spaced along lines of constant lattice constant, ${ }^{5}$ however, this relies on symmetries in the design to achieve crystallinity. For GRIN designs, it is possible to vary the size of inclusions at each grid point on a regular grid to achieve the desired index ${ }^{12}$ but for IR applications this significantly complicates processing. Furthermore, the minimum realizable inclusion diameter is limited, meaning indices between that provided

${ }^{\text {a)} E l e c t r o n i c ~ m a i l: ~ j o h n . h u n t @ d u k e . e d u . ~}$ by the minimum inclusion size and the substrate/air index cannot be achieved. Here we propose a method to generate a hexagonal semicrystalline distribution of uniformly sized inclusions with an arbitrarily varying lattice constant (Fig. 1).

In this method, the inclusions are treated as an analogous system of attractive particles of finite size, where the size or interaction length, of each particle is dependent on its position. The system of particles is allowed to dynamically relax from some initial configuration according to interaction rules defined such that the equilibrium arrangement will have the desired lattice constant distribution. This approach similar to variable size vortex-blob modeling of turbulent fluid flow in which the sizes of the vortices vary with position, ${ }^{13,14}$ and to nonuniform mesh generation techniques. ${ }^{15}$

If the potential energy of two particles has a minimum at a separation of the desired lattice constant, then the particles will automatically arrange themselves with as close to hexagonal packing as possible. The potential energy of two particles with position dependent lattice constants $a_{i}\left(\vec{r}_{i}\right)$ and $a_{j}\left(\vec{r}_{j}\right)$, separated by $R_{i j}=\left|\vec{r}_{i}-\vec{r}_{j}\right|$ is given by,
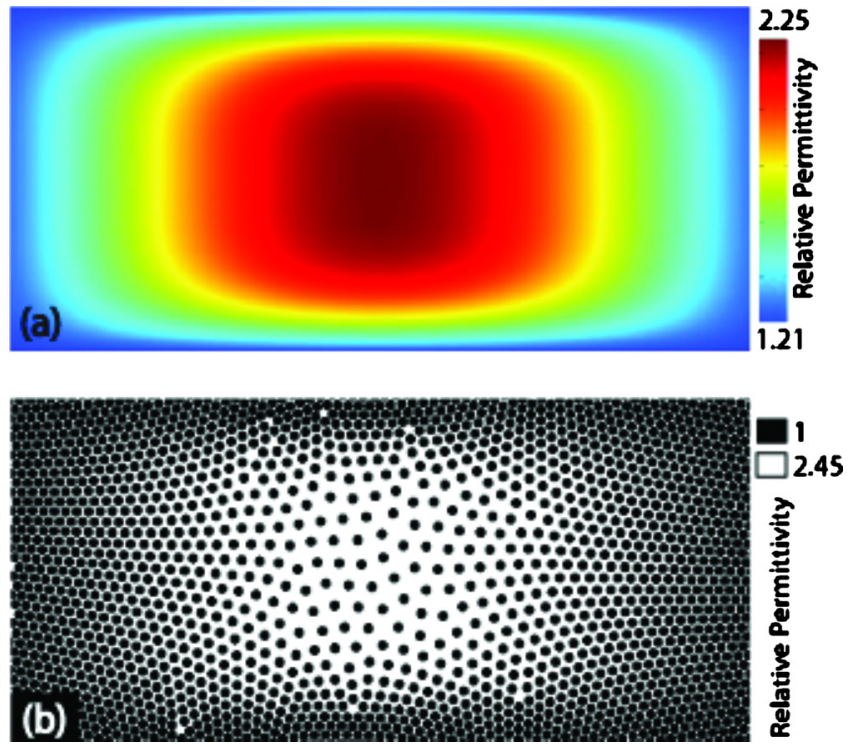

FIG. 1. (Color online) (a) The continuous permittivity map for the 2D TE GRIN lens. (b) The corresponding hole distribution. 


$$
U_{i j}=R_{i j}^{-\alpha}-C_{i j} R_{i j}^{-\beta}, \quad \alpha>\beta,
$$

where,

$$
C_{i j}=\frac{\alpha}{\beta}\left[\frac{a_{i}\left(\vec{r}_{i}\right)+a_{j}\left(\vec{r}_{j}\right)}{2}\right]^{(\beta-\alpha)},
$$

such that the potential energy is minimized at a separation equal to the average of $a_{i}\left(\vec{r}_{i}\right)$ and $a_{j}\left(\vec{r}_{j}\right)$. If the potential well is large (more than $2 a$ ) then the attraction from distant particles will cause the local lattice constant to be less than the ideal. This can be prevented by making the interaction range short by increasing $\beta$ in Eq. (1) and/or setting $U_{i j}$ to a constant for $R_{i j}>\gamma\left(a_{i}+a_{j}\right) / 2$ where $1<\gamma<2$. To reach a static equilibrium state, loss must be added to the particles' equation of motion. The equation of motion for particle $p_{i}$ can then be given by,

$$
\begin{aligned}
& \frac{d^{2} \vec{r}_{i}}{d t^{2}}=\frac{1}{m} \vec{F}_{i}-k \frac{d \vec{r}_{i}}{d t}, \\
& \vec{F}_{i}=\left(1-\delta_{i j}\right) \sum_{j=1}^{N}-\nabla U_{i j},
\end{aligned}
$$

where $m$ is the mass of a particle, $k$ is a drag coefficient, and $N$ is the number of particles in the system. The requirement for equilibrium is that the gradient of $a$ over a unit cell should never be greater than one, as no equilibrium state will exist. Increasing alpha and beta sharpens the potential well resulting in faster convergence with higher positional accuracy. However, if these parameters are set too high the forces on each particle become large enough that the simulation becomes unstable. This instability can be counteracted by increasing the drag coefficient which slows the convergence but maintains the increased positional accuracy.

The relaxation of the lattice can be quantified by calculating the average energy per particle from Eq. (1) for each time step. This energy decreases until some nonzero local minimum is reached at which point the particles have reached an arrangement close to the ideal lattice constant distribution.

The system is initialized with a uniform hexagonal distribution of particles, as this reduces the time-to-equilibrium and the number of defects in the final lattice. Calculating the separation and resulting force between all particles every iteration requires a large amount of computation time, much of which is unnecessary because we require the force between distant particles to be negligible or zero. This computational overhead can be decreased by maintaining a list of each particle's nearest neighbors and only calculating the interaction between these particles. As the particles move or leave the domain, this list must be resorted. However, if the list of nearest neighbors is sufficiently large, then the list can be resorted infrequently. In addition, these resorts can be frontloaded since the degree of particle rearrangement decreases as the system reaches equilibrium.

To create an inclusion distribution with a desired index of refraction profile, we must first generate a lattice constant distribution that will produce the desired index profile. This is done by using an appropriate effective medium approximation to convert our index profile to a lattice constant profile. For instance, for a transverse electric (TE) wave where the electric field is parallel to the inclusion axes, the effective

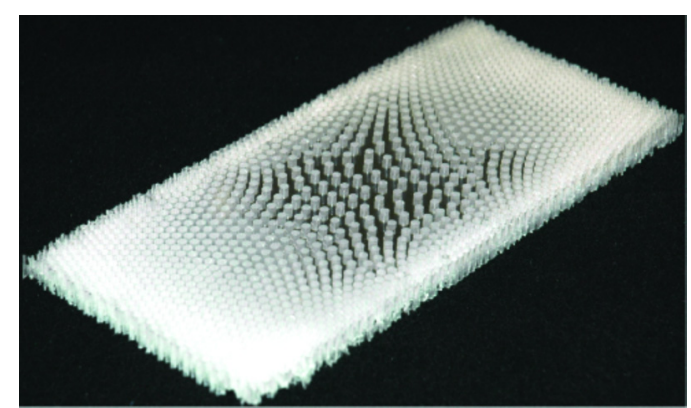

FIG. 2. (Color online) The fabricated polycarbonate lens. The lens size is $0.220 \times 0.1016 \times 0.01 \mathrm{~m}^{3}$ and the hole diameter is $3 \mathrm{~mm}$.

permittivity is determined by the volume average of the matrix permittivity and the inclusion permittivity. The volume fraction of inclusions, $f_{\mathrm{TE}}$, needed to achieve an effective permittivity of $\epsilon_{\mathrm{eff}}$ is then given by,

$$
f_{\mathrm{TE}}=\frac{\epsilon_{\mathrm{eff}}-\epsilon_{\mathrm{matrix}}}{\epsilon_{\mathrm{inc}}-\epsilon_{\mathrm{matrix}}} .
$$

For transverse magnetic (TM) waves where the electric field is transverse to the inclusion axes and the height of the inclusions is much greater than their diameter, the MaxwellGarrnet equation is a better approximation of the effective permittivity. The volume fraction, $f_{\mathrm{TM}}$, is then given by, ${ }^{1}$

$$
f_{\mathrm{TM}}=\frac{\left(\epsilon_{\text {inc }}+\epsilon_{\text {matrix }}\right)\left(\epsilon_{\mathrm{eff}}-\epsilon_{\text {matrix }}\right)}{\left(\epsilon_{\text {inc }}-\epsilon_{\text {matrix }}\right)\left(\epsilon_{\text {eff }}+\epsilon_{\text {matrix }}\right)} .
$$

When the height of the inclusions is comparable to their diameter, then a modification of Eq. (5) accounting for depolarization is required. ${ }^{1}$

The lattice constant, $a$, of a hexagonal lattice with volume fraction, $f$, of inclusions with diameter $d$, is,

$$
\frac{a}{d}=\left(\frac{\pi}{2 \sqrt{3} f}\right)^{1 / 2}, \quad f \leq \frac{\pi}{2 \sqrt{3}} .
$$

The restriction on the domain of $f$ is due to inclusion percolation. Using Eq. (4) or Eqs. (5) and (6) gives an index profile which will have the desired effective index.

The effective medium approximation puts a restriction on the maximum size of the inclusions that can be used. To remain in the homogenizable limit, the maximum lattice constant should be less than or equal to the minimum wavelength of operation divided by ten. For TE operation with hole type inclusions this gives the condition,

$$
d \leq \frac{\lambda}{10}\left(\frac{\pi}{2 \sqrt{3}} \frac{\epsilon_{\text {hole }}-\epsilon_{\text {matrix }}}{\epsilon_{\text {max }}-\epsilon_{\text {matrix }}}\right)^{-1 / 2},
$$

where $\epsilon_{\max }$ is the maximum permittivity required by the design. For column type inclusions the result is the same except that the maximum column size corresponds to the minimum required permittivity.

We have applied this technique to design a 2D microwave GRIN lens using air holes drilled in a polycarbonate slab (Fig. 2). In a simple quartic GRIN lens, the index is constant parallel to the optical axis so reflections occur at the interfaces. Our lens's index profile was designed by modifying a quartic GRIN lens to have an index variation along the optical axis so that the interfaces are nearly impedance matched to air, thus minimizing reflections. The index profile 


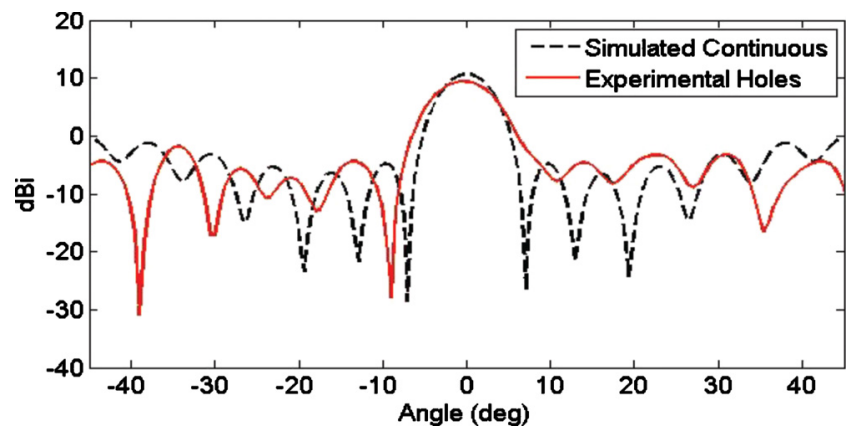

FIG. 3. (Color online) Far-field radiation patterns for the simulated continuous index lens and the experimental holes-only lens at $10 \mathrm{GHz}$ and $0^{\circ}$ incidence.

of the matched GRIN lens was determined by using COMSOL OPTIMIZATION LABORATORY to maximize the far-field intensity of a plane wave produced by a point source located on the optical axis. A $2.1 \mathrm{~dB}$ increase in the far-field intensity compared to the unmatched lens was obtained. The maximum achievable index was determined by Eq. (7) and our minimum machinable hole size, while the minimum achievable index was limited by the percolation condition of Eq. (6).

Figure 1 shows the relative permittivity profile for the matched lens, and the resulting hole distribution. The near-field distribution was measured using a $2 \mathrm{D}$ electric field mapper. ${ }^{16}$ The performance of the lens was then quantified by calculating the far-field pattern of the lens using a 2D Kirchhoff integral and comparing it to the simulated continuous lens, as shown in Fig. 3. The experimental lens exhibits a gain of $9.4 \mathrm{dBi}$ and a beam full-width-at-half-max (FWHM) of $7.2^{\circ}$, while the simulated lens has a gain of 10.6 $\mathrm{dBi}$ and a FWHM of $6.2^{\circ}$.

In conclusion, we have described a technique to generate semicrystalline distributions of particles with arbitrary lattice constant distributions. This method has applications in GRIN devices and PCs, and is particularly suited to the design of IR and visible wavelength devices. We have used this technique to design and implement a microwave GRIN metama- terial lens composed only of uniformly sized holes drilled in a dielectric slab. This binary metamaterial lens shows performance very close to that of the simulated continuous-index lens. This method has also been applied to other more complex lattice constant profiles with multiple maxima and minima and with more complicated domain boundaries. Convergence has been observed in all cases, subject to the previously mentioned constraint on the gradient of $a$. It should be noted that, while we have described and demonstrated this technique for 2D systems, it is readily generalizable to three dimensions.

This work was supported through a Multidisciplinary University Research Initiative, sponsored by the Army Research Office (Contract No. W911NF-09-1-0539). N. Kundtz would like to acknowledge the IC postdoctoral fellowship program.

${ }^{1}$ S. Luryi and A. V. Subashiev, Proc. SPIE 6127, 1 (2006).

${ }^{2}$ N. Grigoropoulos and P. R. Young, Proceedings of the 33rd European Microwave Conference, 2003, p. 439.

${ }^{3}$ T. Zentgraf, G. Bartal, J. Valentine, J. Li, and X. Zhang, Nature Mater. 8, 568 (2009).

${ }^{4}$ C. B. Poitras, L. H. Gabriell, J. Cardenas, and M. Lipson, Nat. Photonics 3, 461 (2009).

${ }^{5}$ L. Xue and V. F. Fusco, IET Proc. Microwaves, Antennas Propag. 1, 624 (2007).

${ }^{6}$ K. Inoshita, T. Baba, D. Mori, and Y. Kuroki, IEEE J. Sel. Top. Quantum Electron. 10, 484 (2004)

${ }^{7}$ Z. Wang, J. Li, L. Xue, and Y. Han, Colloid Polym. Sci. 285, 1037 (2007).

${ }^{8}$ H. Kurt and D. S. Citrin, Opt. Express 15, 1240 (2007).

${ }^{9}$ C. M. Soukoulis, M. Mulot, S. Anand, A. Talneau, M. Agio, and P. Lalanne, Opt. Lett. 29, 1745 (2004).

${ }^{10}$ D. Mori and T. Baba, Opt. Express 13, 9398 (2005).

${ }^{11}$ S. Iwamoto, Y. Wakayama, A. Tandaechanurat, and Y. Arakawa, Opt. Express 16, 21321 (2008).

${ }^{12}$ J. Bai, Z. L. Mei, and T. J. Cui, J. Phys. D 43, 055404 (2010).

${ }^{13}$ G. H. Cottet, P. Koumoutsakos, and M. L. Ould Salihi, J. Comput. Phys. 162, 164 (2000).

${ }^{14}$ T. Y. Hou, SIAM (Soc. Ind. Appl. Math.) J. Numer. Anal. 27, 1387 (1990).

${ }^{15}$ P.-O. Persson and G. Strang, SIAM Rev. 46, 329 (2004).

${ }^{16}$ B. J. Justice, S. A. Cummer, J. B. Pendry, A. F. Starr, D. Schurig, J. J. Mock, and D. R. Smith, Science 314, 977 (2006). 